\title{
Differentiating Lower Extremity Necrotizing Soft Tissue Infection from Severe Cellulitis by Laboratory Parameters and Relevant History Points
}

\section{Kai-Hsiang Wu \\ Chia-Peng Chang (D)}

Department of Emergency Medicine, Chang Gung Memorial Hospital, Chiayi, Taiwan
Correspondence: Chia-Peng Chang Email giovanni850730@gmail.com
Background: Necrotizing soft tissue infection (NSTI) of the lower extremity (LE) is a rapidly progressing infection that requires early diagnosis and prompt treatment to decrease risks of loss of limb or life. Clinical presentation, particularly of early NSTI, can appear similar to severe cellulitis. The purpose of this study is to identify factors that are associated with NSTI rather than severe cellulitis to differentiate patients with similar clinical presentation.

Methods: This retrospective cohort design study compares patients finally diagnosed with LE NSTI versus those diagnosed with severe cellulitis. Cohorts were matched using the modified Laboratory Risk Indicator for Necrotizing Fasciitis (m-LRINEC) score in the setting of LE soft tissue infection. Laboratory values, vital signs, subjective symptoms, and social factors including substance abuse were recorded. Univariate and multivariate analyses were performed.

Results: Multivariate statistical analysis and clinical interpretation of data identified four factors more associated with a diagnosis of NSTI than severe cellulitis: elevated lactate, a patient-reported history of fever, male gender, and intravenous substance user.

Conclusion: In patients with lower extremity infections, the clinical presentation of NSTI and severe cellulitis may appear similar. In this retrospective cohort of patients matched with m-LRINEC scores, elevated lactate, subjective fever, male gender, and intravenous substance abuser were significantly associated with NSTI rather than severe cellulitis. Further studies of these factors in the clinical setting can help tailor the differential diagnosis in the care of patients with severe lower extremity infections. Matched with m-LRINEC scores, elevated lactate, subjective fever, male gender, and intravenous substance abuser were significantly associated with NSTI rather than severe cellulitis. Further studies of these factors in the clinical setting can help tailor the differential diagnosis in the care of patients with severe lower extremity infections.

Keywords: necrotizing soft tissue infection, cellulitis

\section{Introduction}

Necrotizing fasciitis (NF) is a severe, rapidly progressive disease that is characterized by the infection of subcutaneous tissue and fascia, resulting in extensive fascial necrosis. ${ }^{1}$ Lower extremity (LE) NSTI is a limb- and life-threatening condition. Published mortality rates for LE NSTI range from ten to thirty percent, with reviews and meta-analyses publishing overall mortality rates of about $15-20 \% .^{2-5}$ LE NSTI requires emergent surgical intervention including prompt debridement and intravenous 
antibiotics. Timely LE NSTI care is imperative; it has been shown that mortality rates increase steadily with each twenty-four hour period before the first operative debridement. ${ }^{3}$ The first step in efficiently treating NSTI is diagnosing the condition. ${ }^{6}$ Recognizing NSTI can be challenging, because early symptoms of NSTI, such as swelling, erythema, pain are non-specific and can occur with many different types of infections. ${ }^{7}$ Since delay in NSTI diagnosis contributes to increased mortality, an accurate and efficient diagnosis of NSTI is imperative for successfully treating patients with NSTI. ${ }^{8}$

In 2004, a diagnostic scoring system called the Laboratory Risk Indicators for Necrotizing Fasciitis (LRINEC) score was created for this purpose. ${ }^{9}$ The goal of the LRINEC score was to create a "simple and objective scoring system" based on routine laboratory values that could help distinguish NSTI from other soft tissue infections. However, in the ensuing validity studies, researchers found that the validity of the LRINEC had been overstated, and its sensitivity was $43.2-80.0 \%$ for a score of $\geq 6$ and $28.6-68.4 \%$ for a score of $\geq 8$ in different settings, countries or regions. ${ }^{10-}$ ${ }^{12}$ Some studies even demonstrated it to be non-specific. ${ }^{12,13}$ Some authors have questioned if LRINEC scores added any diagnostic value. ${ }^{12,14-17}$

Wu et al developed a modified Laboratory Risk Indicator for Necrotizing Fasciitis (m-LRINEC) scoring system, which reported a better diagnostic value and published on June 2021. ${ }^{18}$ The goal of this study is to compare a matched retrospective cohort of patients with LE NSTI to those with severe cellulitis. Within these two groups of similar m-LRINEC scores, this study identifies factors associated with NSTI rather than cellulitis to help in the clinical differentiation between these diagnoses that require different treatments.

\section{Materials and Methods}

Under the approval of institutional review board, a retrospective cohort study was conducted. The medical records of patients who met the inclusion criteria of surgically proven NF and who received management between January 2015 and January 2020 in two tertiary hospitals were reviewed. Selected comorbidities and initial laboratory values were extracted through medical chart review.

Sixty consecutive, surgically confirmed cases of LE NSTI were identified and assigned to the case group. A control population of 60 consecutive cases of LE severe cellulitis were also reviewed. The control population was matched to the NSTI cohort through average m-LRINEC scores. Because the average m-LRINEC score for the NSTI group was expectedly elevated, eligibility for the control group included a m-LRINEC score of 8 or greater. The goal of this design was to focus on the cases where it is challenging to clinically differentiate between NSTI and cellulitis. For all included patients, variables collected for data analysis were grouped into one of three categories: laboratory values, vital signs at emergency department (ED) presentation, and qualitative data on symptoms and social factors.

Lab values collected for each patient included m-LRINEC score and its six components high-sensitivity $\mathrm{C}$ reactive protein (HCRP), white blood cell count, hemoglobin, sodium, creatinine, and blood glucose, erythrocyte sedimentation rate, lactate, and international normalized ratio. Vital signs at ED presentation that were collected for each patient include maximum temperature (Tmax), maximum heart rate (HRmax), maximum respiratory rate (RRmax), maximum systolic blood pressure (SBPmax), minimum systolic blood pressure (SBPmin), maximum diastolic blood pressure (DBPmax), minimum diastolic blood pressure (DBPmin), maximum mean arterial pressure (MAPmax), minimum mean arterial blood pressure (MAPmin), change in systolic blood pressure (dSBP), change in mean arterial pressure (dMAP), maximum pulse pressure (PPmax), and minimum pulse pressure (PPmin). Additional identifying information, including age and time to presentation, were also grouped into this category. The patient and infection characteristic variables recorded for each case include patient gender, laterality of injury (right or left), homelessness, patient provided history of recent intravenous substance use, subjective fever, and objective fever.

For our statistical analyses, all continuous variables were compared with Mann-Whitney U, whereas categorical variables were compared with chi-square test or Fisher exact test (for expected values less than 5). A binary logistic regression for continuous and categorical variables was also performed. Significance was set at $p<0.05$. Univariate and multivariate analysis was performed.

\section{Results}

In analysis of the quantitative date: lab results and vital signs, univariate and multivariate analyses were performed. Univariate analysis of lab values identified statistically significant differences in two variables - WBC and lactate (Table 1). WBC values were significantly higher in the cohort of patients with NSTI $(21.2 \pm 9.4)$ compared to the cohort with cellulitis $(15.2 \pm 6.3)(\mathrm{p}=0.02)$. Lactate levels were significantly higher among NSTI patients $(18.3 \pm 10.5)$ than 
Table I Laboratory Values from NF and Control Group are Compared Using a Mann-Whitney U-Test

\begin{tabular}{|c|c|c|c|c|c|}
\hline \multirow[t]{2}{*}{ Continue Variables } & \multicolumn{2}{|c|}{ NF Group $(n=60)$} & \multicolumn{2}{|c|}{ Control Group $(n=60)$} & \multirow[t]{2}{*}{ P-value } \\
\hline & (Mean士SD) & Range & (Mean士SD) & Range & \\
\hline m-LRINEC score & $25.1 \pm 9.8$ & $(10-50)$ & $12 \pm 6.9$ & $(8-35)$ & - \\
\hline Age & $49.1 \pm 13.2$ & $(23-90)$ & $46.2 \pm 13.8$ & $(21-88)$ & 0.17 \\
\hline $\mathrm{HCRP}(\mathrm{mg} / \mathrm{dL})$ & $110.5 \pm 76.3$ & $(28-306)$ & $65.8 \pm 20.5$ & $(15-104)$ & 0.06 \\
\hline WBC $\left(\times 10^{4} / \mathrm{uL}\right)$ & $21.2 \pm 9.4$ & $(6.8-51)$ & $15.2 \pm 6.3$ & $(3.7-25.6)$ & $0.02 *$ \\
\hline Blood glucose(mg/dL) & $214.8 \pm 149.6$ & $(83-968)$ & $172.9 \pm 122.7$ & $(64-985)$ & 0.59 \\
\hline Sodium (mEq/L) & $134.5 \pm 7.1$ & $(115-152)$ & $134.6 \pm 5.6$ & $(118-158)$ & 0.44 \\
\hline Lactate (mg/dL) & $18.3 \pm 10.5$ & $(12-109)$ & $8.6 \pm 7.1$ & $(9-36)$ & $<0.01^{*}$ \\
\hline INR & $1.3 \pm 0.4$ & $(0.7-3.6)$ & $1.1 \pm 0.2$ & $(0.8-1.5)$ & 0.19 \\
\hline ESR & $53.1 \pm 28.5$ & $(14-109)$ & $61.9 \pm 28.6$ & $(8-102)$ & 0.84 \\
\hline Hemoglobin & $12.2 \pm 2.5$ & $(4.8-16.6)$ & $11.4 \pm 2.8$ & $(7.6-16.8)$ & 0.63 \\
\hline Tmax & $37.7 \pm 0.9$ & $(36.5-39.4)$ & $37.5 \pm 0.8$ & $(36.2-39.7)$ & 0.43 \\
\hline Time to presentation (days) & $6.5 \pm 4.2$ & $(0-13)$ & $5.2 \pm 3.6$ & $(0-16)$ & 0.74 \\
\hline
\end{tabular}

Note: $* \mathrm{P}<0.05$

Abbreviations: HCRP, high sensitive C reactive protein; WBC, white blood cell count; INR, international normalized ratio; ESR, erythrocyte sedimentation rate; Tmax, maximal temperature at ED; SD, standard deviation.

cellulitis patients $(8.6 \pm 7.1)(\mathrm{p}<0.01)$. Notably, other components of the m-LRINEC score, such as HCRP, hemoglobin, sodium, creatinine, and blood glucose were not different between the two groups. In the multivariate analysis of these laboratory variables, only elevated lactate remained statistically significant $(\mathrm{OR}=3.87, \mathrm{p}<0.01)$. Univariate analysis of vital signs data identified statistically significant, but clinically insignificant, differences in three variables - SBP max, dSBP and, PPmax (Table 2). In the multivariate analysis, no differences in vital signs were found to be statistically significant.
In the analysis of qualitative data, univariate analysis identified significant differences in four variables - homelessness, IV substance user, subjective fever, and gender (Table 3). Patients with NSTI were significantly more likely to be undomiciled $(16.7 \%)$ than patients with cellulitis (3.3\%) $\left(\chi^{2}=11.32, \mathrm{p}<0.01\right)$. Additionally, patients in the NSTI cohort were more likely to abuse IV drugs $(20.0 \%)$ than patients in the cellulitis cohort $(5.0 \%)(\chi 2=15.05$, $\mathrm{p}<0.01)$. Patients with NSTI diagnosis were more likely to have self-reported a subjective fever $(50.0 \%)$ than patients with cellulitis $(26.7 \%) \quad\left(\chi^{2}=16.62, \mathrm{p}<0.01\right)$. In the

Table 2 Vital Signs from NF and Control Group are Compared Using a Mann-Whitney U-Test

\begin{tabular}{|c|c|c|c|c|c|}
\hline \multirow[t]{2}{*}{ Continue Variables } & \multicolumn{2}{|c|}{ NF Group $(n=60)$} & \multicolumn{2}{|c|}{ Control Group $(n=60)$} & \multirow[t]{2}{*}{ P-value } \\
\hline & (Mean士SD) & Range & (Mean士SD) & Range & \\
\hline HRmax & $111.5 \pm 19.6$ & $(72-150)$ & $103.5 \pm 18.5$ & $(66-140)$ & 0.06 \\
\hline RRmax & $23.0 \pm 5.9$ & $(10-39)$ & $22.1 \pm 12.8$ & $(18-35)$ & 0.25 \\
\hline SBPmax & $130.2 \pm 20.3$ & $(104-198)$ & $150.8 \pm 12.5$ & $(118-194)$ & $0.02 *$ \\
\hline DBPmax & $85.7 \pm 14.4$ & $(46-117)$ & $84.4 \pm 13.6$ & $(54-113)$ & 0.78 \\
\hline MAPmax & $103.5 \pm 14.9$ & $(76-129)$ & $107.0 \pm 13.7$ & $(8 I-136)$ & 0.27 \\
\hline SBPmin & $110.3 \pm 17.1$ & $(70-148)$ & $112.5 \pm 18.6$ & $(64-152)$ & 0.56 \\
\hline DBPmin & $67.5 \pm 12.3$ & $(44-86)$ & $65.8 \pm 12.2$ & $(36-94)$ & 0.97 \\
\hline MAPmin & $82.1 \pm 13.5$ & $(58-112)$ & $82.5 \pm 13.2$ & $(56-110)$ & 0.73 \\
\hline dSBP & $26.8 \pm 19.1$ & $(0-75)$ & $35.9 \pm 14.5$ & $(4-95)$ & $0.04 *$ \\
\hline dMAP & $21.4 \pm 14.7$ & $(0-69)$ & $24.4 \pm 11.2$ & $(3-45)$ & 0.22 \\
\hline PPmax & $53.4 \pm 19.2$ & $(18-88)$ & $63.7 \pm 14.6$ & $(40-86)$ & $<0.01^{*}$ \\
\hline PPmin & $43.8 \pm 11.2$ & $(2 I-74)$ & $44.6 \pm 14.3$ & $(17-69)$ & 0.83 \\
\hline
\end{tabular}

Note: $* \mathrm{P}<0.05$

Abbreviations: HRmax, maximum heart rate in ED; RRmax, maximum respiratory rate in ED; SBPmax, maximum systolic blood pressure in ED; DBPmax, maximum diastolic blood pressure in ED; MAPmax, maximum mean arterial pressure in ED; SBPmin, minimum systolic blood pressure in ED; DBPmin, minimum diastolic blood pressure in ED; MAPmin, minimum mean arterial pressure in ED; dSBP, delta systolic pressure; dMAP, delta mean arterial pressure; PPmax, maximum pulse pressure; PPmin, minimum pulse pressure. 
Table 3 Categorical Variables from NF and Control Group are Compared Using a Chi-Squared Test

\begin{tabular}{|c|c|c|c|c|c|c|}
\hline \multirow[t]{2}{*}{ Categorical Variables } & \multicolumn{2}{|c|}{ NF Group $(n=60)$} & \multicolumn{2}{|c|}{ Control Group $(n=60)$} & \multirow[b]{2}{*}{$\chi^{2}$} & \multirow[t]{2}{*}{ p-value } \\
\hline & (n) & $\%$ & (n) & $\%$ & & \\
\hline Gender & & & & & 6.65 & 0.01 \\
\hline Male & 42 & $70.0 \%$ & 37 & $61.7 \%$ & & \\
\hline Female & 18 & $30.0 \%$ & 23 & $38.3 \%$ & & \\
\hline Laterality & & & & & 0.06 & 0.81 \\
\hline Left & 25 & $41.7 \%$ & 24 & $40.0 \%$ & & \\
\hline Right & 35 & $58.3 \%$ & 36 & $60.0 \%$ & & \\
\hline Homeless & & & & & 11.32 & $<0.01$ \\
\hline Yes & 10 & $16.7 \%$ & 2 & $3.3 \%$ & & \\
\hline No & 50 & $83.3 \%$ & 58 & $96.7 \%$ & & \\
\hline IV substance use & & & & & 15.05 & $<0.01$ \\
\hline Yes & 12 & $20.0 \%$ & 3 & $5.0 \%$ & & \\
\hline No & 48 & $80.0 \%$ & 57 & $95.0 \%$ & & \\
\hline Subjective fever & & & & & 16.62 & $<0.01$ \\
\hline Yes & 30 & $50.0 \%$ & 16 & $26.7 \%$ & & \\
\hline No & 30 & $50.0 \%$ & 44 & $73.3 \%$ & & \\
\hline Objective fever & & & & & 3.44 & 0.07 \\
\hline Yes & 23 & $38.3 \%$ & 26 & $43.3 \%$ & & \\
\hline No & 37 & $61.7 \%$ & 34 & $56.7 \%$ & & \\
\hline
\end{tabular}

Note: Patients with an "objective fever" have a temperature $>37.5$ degrees Celsius.

multivariate analysis, subjective fever $(\mathrm{OR}=13.74$, $\mathrm{p}=0.003)$, IV substance use $(\mathrm{OR}=13.98, \mathrm{p}=0.001)$, and gender $(\mathrm{OR}=15.47, \mathrm{p}=0.016)$ all remained statistically significant (Table 4).

\section{Discussion}

Early diagnosis and surgical debridement of LE NSTI is a critical part of treatment in patients with this limb and lifethreatening condition. It is often challenging to definitively diagnose NSTI when the clinical picture is similar to cellulitis. By creating a matched group of control patients with LE cellulitis with, by design, similar m-LRINEC scores, this research identifies other observable clinical factors associated with NSTI rather than cellulitis. Our results indicate that $\mathrm{WBC}$ and lactate levels differ between patients with NSTI and those with serious cellulitis and abscess. While the NSTI group and control group were initially matched through average m-LRINEC scores, WBC a component of m-LRINEC - still differed significantly between the two groups. Some published studies have linked elevated lactate to NSTI diagnosis, and the clinical use of monitoring lactate levels in critically ill patients is common. ${ }^{19} \mathrm{Wu}$ et al proposed other MLRINEC score which assigned highest score in lactate $>18 \mathrm{mg} / \mathrm{dL}$ (OR 4.49, 95\% CI 2.66-12.5.4). ${ }^{20}$ Daniels et al also raised a lactate-based scoring system due to the lactate values were statistically

Table 4 Binary Logistic Regression Displays All Significant Statistically Variables

\begin{tabular}{|c|c|c|c|c|c|c|c|c|}
\hline \multirow[b]{2}{*}{ Factor } & \multirow[b]{2}{*}{ B } & \multirow[b]{2}{*}{ S.E } & \multirow[b]{2}{*}{ Wald } & \multirow[b]{2}{*}{ df } & \multirow[b]{2}{*}{ Sig. } & \multirow[b]{2}{*}{$\operatorname{Exp}(B)$} & \multicolumn{2}{|c|}{$95 \% \mathrm{Cl}$ for $\operatorname{Exp}(\mathrm{B})$} \\
\hline & & & & & & & Lower & Upper \\
\hline Gender & $2.7 I$ & 1.15 & 5.69 & 1.00 & 0.016 & 15.47 & 1.63 & 98.95 \\
\hline Subjective fever & 2.61 & 0.87 & 9.12 & 1.00 & 0.003 & 13.74 & 0.014 & 0.478 \\
\hline IV substance user & 2.63 & 0.82 & 10.38 & 1.00 & 0.001 & 13.98 & 2.95 & 58.41 \\
\hline Lactate & $\mathrm{I} .54$ & 0.58 & 7.34 & 1.00 & 0.006 & 4.970 & 1.52 & 13.85 \\
\hline Constant & -2.28 & 1.27 & 3.22 & 1.00 & 0.078 & 0.152 & - & - \\
\hline
\end{tabular}


significant higher in necrotizing infection group 4.1 vs 2.0 $\mathrm{mmol} / \mathrm{l}(\mathrm{p}<0.001){ }^{21}$ Their finding was similar with our study in the role of elevated lactate. Lactate is a quick, reliable predictor of morbidity and mortality. Additionally, lactate monitoring has been used successfully in riskstratification for critically ill patients. ${ }^{19,22}$ Specifically in regards to NSTI infections, lactate monitoring has been used in multiple studies as a predictor of NSTI mortality rates. ${ }^{23,24}$ Although patients with cellulitis may appear clinically similar at presentation to those with NSTI, the association of higher WBC and higher lactate levels indicate a higher risk to limb and life in patients with LE NSTI. Our results did not find a statistically or clinically meaningful difference in vital signs on initial presentation between the cellulitis group and the NSTI group. Both groups, on average, were tachycardic on hospital presentation. The cause of tachycardia is likely multifactorial. Although it was not clinically or statistically different between the two groups, it is important to record in all patients who present with serious LE infection. In the literature, tachycardia is described as a symptom of NSTI and, in some studies, it has been linked to increased morality rate in patients being treated for NSTI. ${ }^{25,26}$

Although SBP max, dSBP, PPmax were found to be statistically significant in univariate analysis, the differences were not found to be statistically significant in multivariate analysis. Moreover, we do not find the differences in the values to be clinically significant. Analysis of our data indicates that social factors, including housing status and IV substance abuse, are more associated with LE NSTI versus cellulitis of the LE. A strong association between homelessness and LE NSTI was found in both univariate and multivariate analysis. There is limited data in the current literature linking homelessness to NSTI infection. There are epidemiological studies describing outbreaks of Group A Streptococcal (GAS) infection - a cause of both NSTI and cellulitis among a homeless population with incidence of up to 53 times the domiciled population. ${ }^{27,28}$ However, our study is the first to report a statistically significant association between IV substance use and diagnoses of NSTI as opposed to other serious LE soft tissue infections. Though both study populations contained a majority of male patients, gender showed statistical significance in both univariate and multivariate analysis. The group of patients with NSTI was overwhelmingly male compared to the group of patients with cellulitis. This finding is consistent with prior studies reporting higher rates of both cellulitis and NSTI in male patients. ${ }^{29-31}$ However, the clinical significance of this statistical finding is limited, because both groups with soft tissue LE infections show a significant association with male gender.

An additional statistically significant variable found in the analysis of our data is a history of subjective fever. Although subjective fever was more associated with NSTI versus cellulitis, measured temperature and the presence of an objective fever in the emergency department were not significantly associated with one diagnostic group on either univariate or multivariate analysis. Subjective reports of symptoms have been linked to NSTI diagnosis previously, but this is the first association between LE NSTI and subjective fever. Subjectively reported fever may indicate a history of fever since the condition developing from the beginning. Objectively measured fever may be affected by anti-pyretic medication and self-regulation. Subjective fever may reflect more exactly of clinical condition. There are several weaknesses of this study. Firstly, this is a retrospective study that may not be generalizable to other patient populations. In our patient population, LE NSTI seems to disproportionately affect an under researched and under-served population of individuals with a high rate of IV substance abuse and homelessness. Pain out of proportion was mentioned in improving a clinical score for NSTI, ${ }^{32}$ however, level of pain could not be replicable in our retrospective setting. Future studies to early diagnosis of NSTI versus cellulitis should be prospective and, instead of using m-LRINEC scores to match a cohort, could identify patients on clinical presentation where the diagnosis of NSTI versus cellulitis is in question.

\section{Conclusions}

In conclusion, swift and reliable differentiation of LE NSTI from severe cellulitis is of vital clinical importance. This may lead to a limb or life-saving surgical intervention. It is often clinically challenging to accurately differentiate between these illnesses. In cases where the clinical and laboratory evaluation may not give a clear diagnosis, an elevated WBC, lactate, IV substance abuse, and subjective fever should lead clinicians to an increased consideration of LE NSTI for their diagnosis.

\section{Abbreviations}

$\mathrm{CI}$, confidence interval; HCRP, high sensitive C-reactive protein; ED, emergency department; Hb, hemoglobin; LE, low extremity; LRINEC, laboratory risk indicator for necrotizing fasciitis; NSTI, necrotizing soft tissue infection; SD, standard deviation; WBC, white blood cell; INR, international normalized ratio; ESR, erythrocyte sedimentation rate. 


\section{Data Sharing Statement}

All the data will be available upon motivated request to the corresponding author of the present paper.

\section{Ethics Approval and Consent to Participate}

The study was conducted in accordance with the Declaration of Helsinki and the institutional review board of Chiayi Chang Gung Memorial Hospital approved this retrospective study (100-4178B) and (201900447B0C601). Consent to participate was not required. The IRB confirm that the data was anonymized or maintained with confidentiality.

\section{Acknowledgment}

The authors thank all the participants who participated in this study.

\section{Funding}

The study was supported by Chang Gung Memorial Hospital. (CORPG6E0043) (CMRPG6J0111)

(CMRPG6J0112).

\section{Disclosure}

The authors declare that they have no competing interests.

\section{References}

1. Wong C-H, Chang H-C, Pasupathy S, Khin L-W, Tan J-L, Low C-O. Necrotizing fasciitis: clinical presentation, microbiology, and determinants of mortality. J Bone Joint Surg Am. 2003;85-A:1454-60.

2. Anaya DA, McMahon K, Nathens AB, Sullivan SR, Foy H, Bulger E. Predictors of mortality and limb loss in necrotizing soft tissue infections. Arch Surg Chic Ill 1960. 2005;140(2):151-157; discussion 158. doi:10.1001/archsurg.140.2.151

3. Wong C-H, Chang H-C, Pasupathy S, Khin L-W, Tan J-L, Low C-O. Necrotizing fasciitis: clinical presentation, microbiology, and determinants of mortality. J Bone Joint Surg Am. 2003;85(8):1454-1460. doi:10.2106/00004623-200308000-00005

4. Majeski JA, Alexander JW. Early diagnosis, nutritional support, and immediate extensive debridement improve survival in necrotizing fasciitis. Am $J$ Surg. 1983;145(6):784-787. doi:10.1016/00029610(83)90140-X

5. Lee A, May A, Obremskey WT. Necrotizing soft-tissue infections: an orthopaedic emergency. J Am Acad Orthop Surg. 2019;27(5):e199e206. doi:10.5435/JAAOS-D-17-00616

6. Anaya DA, Dellinger EP. Necrotizing soft-tissue infection: diagnosis and management. Clin Infect Dis. 2007;44(5):705-710. doi:10.1086/ 511638

7. Avalahalli MG, Muniraja PK, Khalid MS, Kaverappa K, Devaraj L, Rao A. Comparative study of LRINEC score: procalcitonin and lrinec score: c-reactive protein in predicting duration of hospital stay and severity in necrotizing fasciitis. J Evol Med Dent Sci. 2016;5(51):3248. doi: $10.14260 /$ jemds/2016/754

8. Taviloglu K, Yanar H. Necrotizing fasciitis: strategies for diagnosis and management. World J Emerg Surg WJES. 2007;2(1):19. doi:10.1186/1749-7922-2-19
9. Wong C-H, Khin L-W, Heng K-S, Tan K-C, Low C-O. The LRINEC (laboratory risk indicator for necrotizing fasciitis) score: a tool for distinguishing necrotizing fasciitis from other soft tissue infections. Crit Care Med. 2004;32(7):1535-1541. doi:10.1097/01. ccm.0000129486.35458.7d

10. Tan J, Koh B, Hong C, et al. A comparison of necrotising fasciitis in diabetics and non-diabetics: a review of 127 patients. Bone Joint J. 2016;11(11):1563-1568. doi:10.1302/0301-620X.98B11.37526

11. Holland MJ. Application of the Laboratory Risk Indicator in Necrotising Fasciitis (LRINEC) score to patients in a tropical tertiary referral centre. Anaesth Intensive Care. 2009;37(4):588-592. doi:10.1177/0310057X0903700416

12. Abdullah M, McWilliams B, Khan SU. Reliability of the Laboratory Risk Indicator in Necrotising Fasciitis (LRINEC) score. Surgeon. 2019;17(5):309-318. doi:10.1016/j.surge.2018.08.001

13. Johnson L, Crisologo P, Sivaganesan S, Caldwell C, Henning J. Evaluation of the Laboratory Risk Indicator for Necrotizing Fasciitis (LRINEC) score for detecting necrotizing soft tissue infections in patients with diabetes and lower extremity infection. Diabetes Res Clin Pract. 2020;171:108520. doi:10.1016/j. diabres.2020.108520

14. Hansen MB, Rasmussen LS, Svensson M, et al. Association between cytokine response, the LRINEC score and outcome in patients with necrotizing soft tissue infection: a Multicentre, Prospective Study. Sci Rep. 2017;7(1):42179. doi:10.1038/srep42179

15. Wiggenhauser PS, Hoffmann TK. Comment on: "cervical necrotizing fasciitis - the value of the laboratory risk indicator for necrotizing fasciitis score as an indicative parameter. J Oral Maxillofac Surg. 2016;74(6):1104-1105. doi:10.1016/j.joms.2015.12.025

16. Liao C-I, Lee Y-K, Su Y-C, Chuang C-H, Wong C-H. Validation of the laboratory risk indicator for necrotizing fasciitis (LRINEC) score for early diagnosis of necrotizing fasciitis. Tzu Chi Med J. 2012;24 (2):73-76. doi:10.1016/j.tcmj.2012.02.009

17. Hsiao CT, Chang CP, Huang TY, Chen YC, Fann WC. Prospective validation of the Laboratory Risk Indicator for Necrotizing Fasciitis (LRINEC) score for necrotizing fasciitis of the extremities. PLoS One. 2020;15(1):e227748. doi:10.1371/journal.pone.0227748

18. Wu H, Liu S, Li C, Song Z. Modified Laboratory Risk Indicator for Necrotizing Fasciitis (m-LRINEC) score system in diagnosing necrotizing fasciitis: a Nested Case-Control Study. Infect Drug Resist. 2021;14:2105-2112. doi:10.2147/IDR.S313321

19. Bakker J, Nijsten MW, Jansen TC. Clinical use of lactate monitoring in critically ill patients. Ann Intensive Care. 2013;3(1):12. doi:10.1186/2110-5820-3-12

20. Wu P-H, Wu K-H, Hsiao C-T, Wu S-R, Chang C-P. Utility of modified Laboratory Risk Indicator for Necrotizing Fasciitis (MLRINEC) score in distinguishing necrotizing from non-necrotizing soft tissue infections. World J Emerg Surg. 2021;16 (1):26. doi:10.1186/s13017-021-00373-0

21. Daniels M, Oberländer H, Schiefer J, et al. Lactate based scoring system in the diagnosis of necrotizing fasciitis. J Burn Care Res. 2021;irab148. doi:10.1093/jbcr/irab148

22. Jansen TC, van Bommel J, Bakker J. Blood lactate monitoring in critically ill patients: a systematic health technology assessment. Crit Care Med. 2009;37(10):2827-2839. doi:10.1097/ CCM.0b013e3181a98899

23. Chang C-P, Fann W-C, Wu S-R, Lin C-N, Hsiao C-T. Lactate on emergency department arrival as a predictor of in-hospital mortality in necrotizing fasciitis: a retrospective study. J Orthop Surg. 2019;14 (1):73. doi:10.1186/s13018-019-1108-y

24. Yaghoubian A, de Virgilio C, Dauphine C, Lewis RJ, Lin M. Use of admission serum lactate and sodium levels to predict mortality in necrotizing soft-tissue infections. Arch Surg. 2007;142(9):840-846. doi:10.1001/archsurg. 142.9.840

25. Wallace HA, Waheed A, Perera TB. Necrotizing fasciitis. In: StatPearls. Treasure Island (FL): StatPearls Publishing; 2019. 
26. Dworkin MS, Westercamp MD, Park L, Mcintyre A. The epidemiology of necrotizing fasciitis including factors associated with death and amputation. Epidemiol Infect. 2009;137(11):1609-1614. doi:10.1017/S0950268809002532

27. Khamnuan P, Chongruksut W, Jearwattanakanok K, Patumanond J, Yodluangfun S, Tantraworasin A. Necrotizing fasciitis: risk factors of mortality. Risk Manag Healthc Policy. 2015;8:1-7. doi:10.2147/ RMHP.S77691

28. Mosites E, Zulz T, Bruden D, et al. Risk for Invasive streptococcal infections among adults experiencing homelessness, Anchorage, Alaska, USA, 2002-2015. Emerg Infect Dis. 2019;25 (10):1911-1918. doi:10.3201/eid2510.181408

29. Bruun T, Oppegaard O, Kittang BR, Mylvaganam H, Langeland N, Skrede S. Etiology of cellulitis and clinical prediction of streptococcal disease: a Prospective Study. Open Forum Infect Dis. 2015;3:1. doi:10.1093/ofid/ofv181
30. Ellis Simonsen SM, Van Orman ER, Hatch BE, et al. Cellulitis incidence in a defined population. Epidemiol Infect. 2006;134 (2):293-299. doi:10.1017/S095026880500484X

31. Misiakos EP, Bagias G, Patapis P, Sotiropoulos D, Kanavidis P, Machairas A. Current concepts in the management of necrotizing fasciitis. Front Surg. 2014;1:36. doi:10.3389/fsurg.2014.00036

32. Borschitz T, Schlicht S, Siegel E, Hanke E, von Stebut E. Improvement of a clinical score for necrotizing fasciitis: 'pain out of proportion' and high CRP levels aid the diagnosis. PLoS One. 2015;10(7):e0132775. doi:10.1371/journal.pone.0132775

\section{Publish your work in this journal}

Infection and Drug Resistance is an international, peer-reviewed openaccess journal that focuses on the optimal treatment of infection (bacterial, fungal and viral) and the development and institution of preventive strategies to minimize the development and spread of resistance. The journal is specifically concerned with the epidemiology of antibiotic resistance and the mechanisms of resistance development and diffusion in both hospitals and the community. The manuscript management system is completely online and includes a very quick and fair peerreview system, which is all easy to use. Visit http://www.dovepress.com/ testimonials.php to read real quotes from published authors. 